\title{
Changing trends in clinico-pathologic characteristics and treatment outcomes in patients with gastric cancer: A single-center, public hospital, retrospective study
}

\author{
Min-Seung Gwak, Jong-Min Park \\ Department of Surgery, National Medical Center, Seoul, Korea
}

\begin{abstract}
Purpose: We analyzed our 10-year experience in a single-center, public hospital and thereby evaluated the changing trends of clinico-pathologic and surgical characteristics as well as treatment outcomes in patients with gastric cancer.

Methods: The current single-center, retrospective study was conducted with patients who had been treated at department of our medical institution during a period ranging from March 1, 2007 to June 16, 2018. The eligible patients were divided into two groups: group I (March 2007-April 2012) and II (May 2012-June 2018). Then, we compared time-dependent changes in clinico-pathologic characteristics between the two groups.

Results: The mean age was $63.0 \pm 11.3$ years in group I and $65.8 \pm 10.5$ years in group II, respectively ( $P=0.017)$. The American Society of Anesthesiologist (ASA) score was 34.9\% for 1 point, 38.3\% for 2 points, and 26.9\% for 3 points or more in group I, and 31.1\% for 1 point, $52.5 \%$ for 2 points, and $16.4 \%$ for 3 points or more in group $I I$, which was statistically significant $(P=0.012)$. The average follow-up duration was significantly different between the two group $(39.8 \pm 39.7$ vs. $23.4 \pm 20.6)(P<0.001)$. The duration of postoperative hospital stay was 1.8 days longer in group II than group I ( $\mathrm{P}=0.047)$. Tumor depth, node metastasis and distant metastasis were significantly different between the two groups $(P<0.001, P=0.009$, and $P=0.019$, respectively).

Conclusion: There were significant differences in the age, ASA score, average follow-up duration, postoperative hospital stay, tumor depth, node metastasis and distant metastasis between the two groups.
\end{abstract}

Keywords: Stomach neoplasms, Gastrectomy, Treatment outcome, Public hospital

\section{INTRODUCTION}

Gastric cancer (GC) is known as the 5th most common malignancy, and it is also the 3 rd leading cause of death worldwide [1]. Despite a recent decline in its incidence in developed countries, 1.3

Received: Aug 30, 2018 Revised: Nov 09, 2018 Accepted: Nov 29, 2018 Correspondence to: Jong-Min Park

Department of Surgery, National Medical Center, 245 Eulji-ro, Jung-gu, Seoul 04564, Korea

Tel: +82-2-2260-4883, Fax: +82-2-2269-0750

E-mail:jmparkgs@gmail.com

ORCID: Min-Seung Gwak (https://orcid.org/0000-0003-4440-8537), Jong-Min Park (http://orcid.org/0000-0003-4023-5560)

Copyright (C) 2018 Korean Society of Surgical Oncology

This is an Open Access article distributed under the terms of the Creative Commons Attribution Non-Commercial License (http://creativecommons.org/licenses/by-nc/4.0) which permits unrestricted non-commercial use, distribution, and reproduction in any medium, provided the original work is properly cited. million patients are annually diagnosed with GC. This is also accompanied by a report of 819,000 cases of deaths in 2015 [2]. In Korea, GC is the 2nd most prevalent cancer and the 3rd leading cause of death [3].

Despite considerable advancements in outcomes of GC treatment, accompanied by improved prognosis, which are attributable to early diagnosis, radical operation and development of adjuvant therapies, its poor prognosis remains worrisome [4].

Over several decades, there have been great advancements in treatment modalities and strategies for patients with GC. This is accompanied by recent trends in which treatment approaches have been customized to both patient and tumor characteristics [5]. Furthermore, there have been dramatic changes in treatment strategy, which is mainly due to advances in chemotherapy. Still, however, curative surgery remains a main stay of treatment for patients with GC. There is a variability in the survival rate depending on 
several factors in patients receiving curative surgery. Of these, the stage of disease and the quality of surgery serve as important predictors of patient survival [6]. It has been reported that treatment outcomes are the most excellent in patients with limited tumor invasion [7]. This is accompanied by published studies showing that the 5-year overall survival is at its highest; up to $99 \%$ and $66 \%$ in patients with early GC (pT1 and pT2, respectively) [8]. Thus, increased survival has arisen from extensive lymph node dissection combined with a resection of adjacent organs and the improvement of operative care, as well as early detection of GC using an advanced endoscopy technique $[9,10]$.

To date, many studies have reported changing trends in the histologic type, location, stage, the incidence of early GC, and postoperative outcomes [11]. A nationwide survey reported the trend of clinico-pathological features of GC patients in Korea [12]. We reported short-term outcomes of GC surgery in a public hospital in a previous study conducted in 2012 [13]. In the study, we showed that the characteristics of our patients differ from those of the entirety of our nations GC patients. The purpose of this study is to investigate the changes in the characteristics of patients with GC in a public hospital.

Given the above background, we analyzed our 10-year experience in a single-center, public hospital thereby evaluating the changing trends of clinico-pathologic and surgical characteristics as well as treatment outcomes.

\section{METHODS}

\section{Study patients and setting}

The current single-center, retrospective study was conducted using patients who had been treated at a department of National Medical Center during a period ranging from March 1, 2007 to June 16, 2018.

Inclusion criteria for the current study are as follows: (1) patients with GC who had undergone gastric surgery; and (2) patients with available data about demographic and clinical characteristics, pathologic findings, operative records, and follow-up results.

Exclusion criteria for the current study are as follows: (1) patients with other gastric malignancies (e.g., such as gastric lymphoma) or synchronous or metachronous primary tumors (e.g., breast cancer, colorectal cancer); (2) patients with inoperable GC (e.g., such as exploratory laparotomy, open \& closure); or (3) patients who were deemed to be ineligible for study participation according to our judgment.

We therefore enrolled a total of 358 patients in the current study; it was approved by the Institutional Review Board (IRB) of National Medical Center (IRB No. H-1807-092-011). Informed consent was waived due to its retrospective nature.

\section{Patient evaluation and criteria}

We made a diagnosis of GC in the patients with a histopathologically proven adenocarcinoma located predominantly in the stomach. For convenience, all the patients were staged based on the 7th edition of the American Joint Committee on Cancer/Union for International Cancer Control (AJCC/UICC) TNM staging system.

We retrospectively analyzed factors that may affect the treatment outcomes; these include demographic characteristics (e.g., age and sex), underlying diseases (e.g., hypertension and diabetes), average follow-up duration, medical insurance type (e.g., health insurance and medical aid), operative factors (e.g., types and methods of surgery, curability, combined resection, postoperative complications, mortality, postoperative length of hospital stay, recurrence and disease-free survival [DFS]) and pathologic findings.

Underlying diseases were defined using the patients who were receiving medical treatments or using healthcare services more than 6 times a year. The physical status of the patients was preoperatively evaluated based on the American Society of Anesthesiologist (ASA) physical status classification system. Complications were defined using the patients who were in need of invasive or non-invasive treatments that could not be performed as a standardized critical pathway for GC surgery.

Combined resection was performed for the patients who were suspected of having an invasion to the adjacent organs (e.g., cholecystectomy and splenectomy).

Treatment outcomes were evaluated based on the 2-year and 5-year DFS; it was defined as the length of period elapsing until the first event occurred since gastric surgery was performed. The event was defined as cases such as the first recurrence and death due to GC. Pathologic outcomes were evaluated based on the 7th AJCC/ UICC TNM staging system for GC.

To examine time trends of clinico-pathologic characteristics depending on the period when they received treatments, we divided our clinical series of the patients into two groups: group I (March 2007-April 2012) and II (May 2012-June 2018). Then, we compared time-dependent changes in clinico-pathologic characteristics between the two groups.

\section{Statistical analysis}

All data was expressed as mean \pm standard deviation. To identify statistical significance, we performed the chi-square test or Fisher exact test. Survival analysis was performed using the Kaplan-Meier method. Statistical analysis was done using the SPSS 17.0 for Windows (SPSS Inc., Chicago, IL, USA). A P-value of $<0.05$ was considered statistically significant. 


\section{RESULTS}

\section{Baseline characteristics of the patients}

The overall patient characteristics are shown in Table 1. From March 2007 to June 2018, 358 patients underwent surgery for GC. The mean age was $64.4 \pm 11.0$ years, with 277 males and $81 \mathrm{fe}$ -

Table 1. Characteristics of the patients

\begin{tabular}{lc}
\hline Variable & Value \\
\hline Age $($ yr) & $64.4 \pm 11.0$ \\
Sex & $277(77.4)$ \\
Male & $81(22.6)$ \\
Female & \\
ASA score & $118(33)$ \\
1 & $163(45.5)$ \\
2 & $77(21.5)$ \\
$\geq 3$ & \\
Co-morbidities & $261(72.9)$ \\
Yes & $97(27.1)$ \\
No & \\
Symptom & $230(64.2)$ \\
Yes & $128(35.8)$ \\
No & $31.4 \pm 32.4$ \\
Follow-up duration (mo) & \\
Medical insurance & $241(67.3)$ \\
Health insurance & $117(32.7)$ \\
Medical aid &
\end{tabular}

Values are presented as mean \pm standard deviation or number $(\%)$. ASA, American Society of Anesthesiologist.

Table 2. Characteristics for the patients of each group

\begin{tabular}{lccc}
\hline Variable & Group I $(\mathrm{n}=175)$ & Group II $(\mathrm{n}=183)$ & P-value \\
\hline Age (yr) & $63.0 \pm 11.3$ & $65.8 \pm 10.5$ & 0.017 \\
Sex & & & 0.172 \\
$\quad$ Male & $130(74.3)$ & $147(80.3)$ & \\
Female & $45(25.7)$ & $36(19.7)$ & \\
ASA score & & & 0.012 \\
1 & $61(34.9)$ & $57(31.1)$ & \\
2 & $67(38.3)$ & $96(52.5)$ & \\
$\geq 3$ & $47(26.9)$ & $30(16.4)$ & \\
Co-morbidities & & & \\
Yes & $127(72.6)$ & $134(73.2)$ & \\
No & $48(27.4)$ & $49(26.8)$ & \\
Symptom & & & \\
Yes & $114(65.1)$ & $116(63.4)$ & \\
No & $61(34.9)$ & $67(36.6)$ & \\
Follow-up duration (mo) & $39.8 \pm 39.7$ & $23.4 \pm 20.6$ & $<0.001$ \\
Medical insurance & & & 0.125 \\
Health insurance & $111(63.4)$ & $130(71)$ & \\
Medical aid & $64(36.6)$ & $53(29)$ & \\
\hline
\end{tabular}

Values are presented as mean \pm standard deviation or number (\%). ASA, American Society of Anesthesiologist. males. The ASA score was $33 \%$ for 1 point, $45.5 \%$ for 2 points, and $77 \%$ for 3 points or higher. Comorbidities were present in $72.9 \%$ and symptoms were experienced in $64.2 \%$. The average follow-up duration was $31.4 \pm 32.4$ months. Of the total patients, 241 (67.3\%) had health insurance and 117 (32.7\%) were medical aid beneficiaries (Table 1).

The patients were divided into group I $(\mathrm{n}=175)$ and group II $(\mathrm{n}=183)$. The mean age was $63.0 \pm 11.3$ years in group I and $65.8 \pm 10.5$ years in group II $(\mathrm{P}=0.017)$. There were no significant differences in the male-to-female ratio between the two groups (130:45 vs. $147: 36)(\mathrm{P}=0.172)$. The ASA score was $34.9 \%$ for 1 point, $38.3 \%$ for 2 points, and $26.9 \%$ for 3 points or more in group I, and $31.1 \%$ for 1 point, $52.5 \%$ for 2 points, and $16.4 \%$ for 3 points or more in group II, which was statistically significant $(\mathrm{P}=0.012)$. There were no significant differences in the co-morbidities and symptoms between the two groups. The average follow-up duration was significantly different between the two groups ( $39.8 \pm 39.7$ vs. $23.4 \pm 20.6)(\mathrm{P}<0.001)$. There were no significant differences in medical insurance type between the two groups $(\mathrm{P}=0.125)$ (Table 2$)$.

Table 3. Surgical and clinical outcomes of each group

\begin{tabular}{|c|c|c|c|}
\hline Variable & Group I $(n=175)$ & Group II $(n=183)$ & P-value \\
\hline Surgery type & & & 0.120 \\
\hline Open STG & $50(28.6)$ & 59 (32.2) & \\
\hline Open TG & 25 (14.3) & $32(17.5)$ & \\
\hline Open others & $13(7.4)$ & $6(3.3)$ & \\
\hline Laparoscopic STG & $63(36)$ & 73 (39.9) & \\
\hline Laparoscopic TG & $15(8.6)$ & $10(5.5)$ & \\
\hline Laparoscopic others & $9(5.1)$ & $3(1.6)$ & \\
\hline Radicality & & & 0.068 \\
\hline Ro & 137 (78.3) & $161(88)$ & \\
\hline R1 & $5(2.9)$ & $5(2.7)$ & \\
\hline R2 & $13(7.4)$ & $8(4.4)$ & \\
\hline No resection & $20(11.4)$ & $9(4.9)$ & \\
\hline Combined operation & & & 0.954 \\
\hline Yes & 55 (31.4) & $57(31.1)$ & \\
\hline No & $120(68.6)$ & $126(68.9)$ & \\
\hline Complication & & & 0.077 \\
\hline Yes & $63(36)$ & $50(27.3)$ & \\
\hline No & 112 (64) & $133(72.7)$ & \\
\hline 30-Day mortality & 9 & 6 & 0.379 \\
\hline $\begin{array}{l}\text { Postoperative hospital stay } \\
\text { (day) }\end{array}$ & $12.6 \pm 8.5$ & $14.4 \pm 8.7$ & 0.047 \\
\hline Recurrence & & & 0.008 \\
\hline Yes & $24(13.7)$ & $10(5.5)$ & \\
\hline No & $151(86.3)$ & $173(94.5)$ & \\
\hline DFS $(\%)$ & & & 0.011 \\
\hline 2 Years & 71.1 & 84.4 & \\
\hline 5 Years & 67.4 & 81.9 & \\
\hline
\end{tabular}

Values are presented as number (\%), mean \pm standard deviation, or number. STG, subtotal gastrectomy; TG, total gastrectomy; DFS, disease-free survival. 


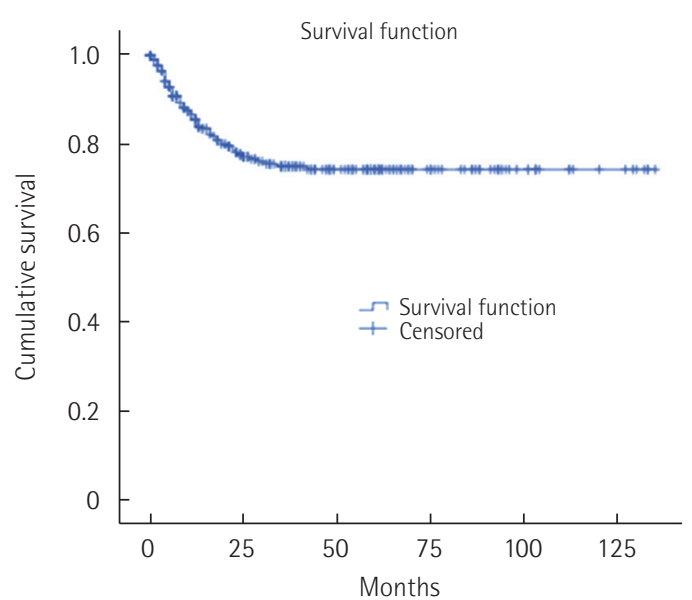

Fig. 1. Disease-free survival curve of total gastric cancer patients.

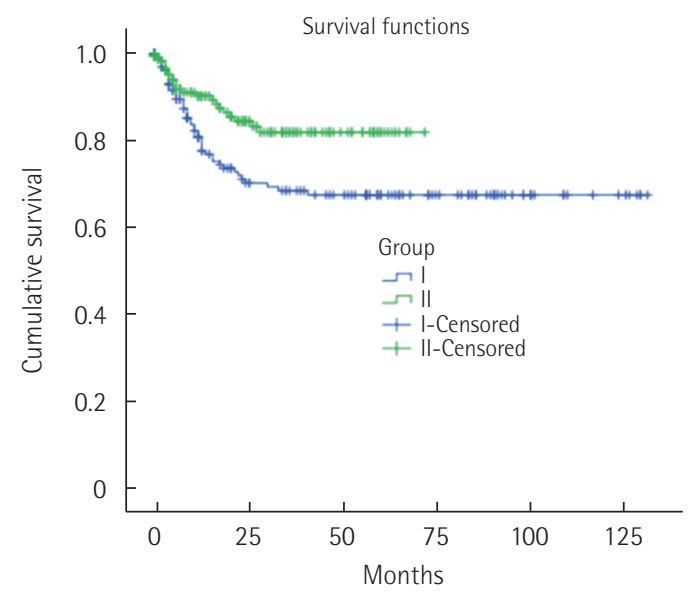

Fig. 2. Disease-free survival curves of gastric cancer patients group I and group II.

\section{Time trends of clinico-pathologic characteristics of the patients}

Our surgical and clinical outcomes are summarized in Table 3. There were no significant differences in the surgery type between the two groups $(\mathrm{P}=0.12)$. Laparoscopic subtotal gastrectomy was the most common procedure in both groups, open subtotal gastrectomy and open total gastrectomy followed. There were no significant differences in the radicality, combined operation, and complication rate. The duration of postoperative hospital stay was 1.8 days longer in group II than in group I $(\mathrm{P}=0.047)$. The recurrence rate was higher in group I (13.7\%) than in group II (5.5\%) $(\mathrm{P}=0.008)$. There were no significant differences in the 30 -day mortality between the two groups ( 9 vs. $6, \mathrm{P}=0.379$ ). The survival curve of the total patients is shown in Fig. 1. The 2-year and 5-year DFS was $77.5 \%$ and $74.2 \%$, respectively. There were significant differences in the survival rate between the two groups $(\mathrm{P}=0.011)$. The 2-year DFS was $71.1 \%$ in group I and $84.4 \%$ in group II. The
Table 4. Pathologic outcomes of each group

\begin{tabular}{|c|c|c|c|}
\hline Variable & Group I $(n=175)$ & Group II $(n=183)$ & P-value \\
\hline Tumor location & & & 0.766 \\
\hline Upper third & $30(17.1)$ & 31 (16.9) & \\
\hline Mid third & $41(23.4)$ & $41(22.4)$ & \\
\hline Low third & $97(55.4)$ & $107(58.5)$ & \\
\hline Entire & $7(4)$ & $4(2.2)$ & \\
\hline Histologic type & & & 0.747 \\
\hline Differentiated & $65(37.1)$ & 71 (38.8) & \\
\hline Undifferentiated & $110(62.9)$ & $112(61.2)$ & \\
\hline Tumor depth & & & $<0.001$ \\
\hline $\mathrm{T} 1$ & $71(40.6)$ & $77(42.1)$ & \\
\hline T2 & 18 (10.3) & $21(11.5)$ & \\
\hline T3 & $6(3.4)$ & $43(23.5)$ & \\
\hline $\mathrm{T} 4$ & $80(45.7)$ & $42(22.9)$ & \\
\hline Node metastasis & & & 0.009 \\
\hline No & 95 (54.3) & $107(58.5)$ & \\
\hline N1 & $17(9.7)$ & $17(9.3)$ & \\
\hline N2 & $11(6.3)$ & $18(9.8)$ & \\
\hline N3 & $25(14.3)$ & $33(18)$ & \\
\hline $\mathrm{Nx}$ & $27(15.4)$ & $8(4.4)$ & \\
\hline Distant metastasis & & & 0.019 \\
\hline Mo & $132(75.4)$ & $156(85.2)$ & \\
\hline M1 & $43(24.6)$ & $27(14.8)$ & \\
\hline Stage $e^{a)}$ & & & 0.164 \\
\hline $\mathrm{IA}$ & $66(37.7)$ & $70(38.5)$ & \\
\hline IB & $14(8)$ & $18(9.8)$ & \\
\hline$\| A$ & $9(5.1)$ & $21(11.5)$ & \\
\hline$\| B$ & $10(5.7)$ & $10(5.5)$ & \\
\hline$\| \mathrm{A}$ & $11(6.3)$ & $11(6)$ & \\
\hline$\| I \mid B$ & $10(5.7)$ & $16(8.7)$ & \\
\hline$\| I I C$ & $12(6.9)$ & $10(5.5)$ & \\
\hline IV & $43(24.6)$ & $27(14.8)$ & \\
\hline
\end{tabular}

Values are presented as number (\%).

a) The 7th edition of the American Joint Committee on Cancer/Union for International Cancer Control TNM staging system.

5-year DFS was 64.7\% in group I and 81.9\% in group II (Table 3). The survival curve of each group is shown in Fig. 2.

Postoperative histopathological findings are summarized in Table 4. The location of the tumor and histologic type was not statistically significant $(\mathrm{P}=0.766$ and $\mathrm{P}=0.747$, respectively). The proportion of $\mathrm{T} 1$ that corresponded to early GC in tumor invasion was $40.6 \%$ in group I and $42.1 \%$ in group II. The total proportion of patients with T2, T3, and T4 that corresponded to advanced GC was similar between the two groups, the proportion of T4 was significantly higher in group I than in group II (10.3:3.4:45.7 vs. 11.5:23.5:22.9) $(\mathrm{P}<0.001)$. Lymph node metastasis and distant metastasis were also statistically significant $(\mathrm{P}=0.009$ and $\mathrm{P}=0.019$, respectively). There was no significant difference in stage $(\mathrm{P}=0.164)$ (Table 4$)$. 


\section{DISCUSSION}

In the past, GC was regarded as a disease entity whose characteristics remained unchanged [14]. Recent studies have revealed, however, that there are time-dependent changes in the clinico-pathologic features, biological characteristics, and disease course of GC $[11,15]$. This is possibly due to technical advancements in the diagnosis and treatment of GC [16]. Moreover, there have been improvements in treatment outcomes in patients with GC with the introduction of advanced treatment modalities; these include types of surgical interventions, peri-operative care, the scope of excision and dissection, surgeons' technical expertise and use of new surgical equipment [17].

There are worldwide trends in which the age of patients with GC increases; it has also been reported to occur most prevalently in patients in their seventies $[18,19]$. Our study also showed an increase in the mean age of 2.8 years in group II. Still, however, the most common age group is in their 60s. There is controversy as to whether age is associated with long-term survival in patients with GC. Based on a previous published study showing that there were significant increases in biological markers indicating malignant behavior and pathologic features in younger patients as compared with older ones, it can be inferred that younger patients are vulnerable to a poorer prognosis [20]. But there is also a controversial report showing that older patients had poor tolerability as well as limited lymph node dissection during chemotherapy [21]. These issues related to age need further study in the future.

It has been reported that the incidence of GC in the upper onethird is significantly higher in Western countries as compared with Asian countries, such as Korea and Japan [22]. Of note, there are also recent trends that the incidence of proximal GC has increased. Presumably, this might be due to alterations in risk factors, such as smoking, alcohol consumption and gastroesophageal reflux disease [23]. Furthermore, it has also been reported that patients with proximal GC had a poorer prognosis [18]. We reported short-term outcomes of GC surgery in a public hospital in 2012 in a previous study. According to a 2014 nationwide survey, the proportion of upper, middle, and lower third GC was $13.4 \%, 28.6 \%$, and $56 \%$, respectively. However, in our 2012 study, the proportion of tumor location was $17.2 \%, 24.2 \%$, and $54.3 \%$, respectively. Moreover, the proportion of T4 tumor, M1 stage, and stage IV was considerably higher in our patients. These patients mainly consisted of medical aid beneficiaries $[12,13]$. Our medical institution had higher upper third GC patients, which was also related to the prognosis of the patient. In our study, there was no significant difference in cancer occurrence location between the two groups. The ratio of upper third GC remained around $17 \%$ as in the previous study. The pa- tients visiting our hospital had a high proportion of upper GC and poor prognosis. The reason this ratio is high in the socioeconomic vulnerable class visiting public hospitals needs further research.

There are also changing trends in the histologic type; the incidence of intestinal type malignancies and diffuse type GC decreased and increased, respectively $[11,15]$. Moreover, poorly-differentiated tumors, showing an increase in their prevalence, had a poorer prognosis as compared with well- or moderately-differentiated ones, which is also accompanied by a report that signet ring cell cancer or mucinous adenocarcinoma had a poorer prognosis as compared with well- or moderately-differentiated ones [24]. In our study, no significant change in histologic type over time was observed between the two groups.

According to the National Cancer Screening Program in Japan and Korea, early GC accounts for $40 \%$ to $60 \%$ of total cases of GC $[19,25]$. There has been an increase in its incidence, which is probably attributable to standardized protocols using diagnostic equipment [26]. This is accompanied by published studies showing that patients with early GC had a better prognosis as compared with those with advanced stage $[18,19,27]$. In our study, the ratio of early GC to advanced GC was not significantly different between the two groups. Although T4 has decreased 22.8\% more in group II than in group I, the proportion of advanced GC still remained at $60 \%$. Considering that public hospitals have a high percentage of medical aid beneficiaries and homeless people, it can be inferred that early screening rates are still low. This is supported by the fact that there was no significant difference in the proportion of health insurance and medical aid patients between the two groups.

Controversial opinions exist regarding the benefit of extended lymph node dissection in patients with GC. But its effects in prolonging survival have been advocated by several prospective randomized controlled trials $[16,28,29]$. Concurrent adjuvant chemotherapies with advanced surgical skills may affect the life expectancy of patients with GC. Moreover, tumor staging as well as the number of retrieved lymph nodes has a positive prognostic value [30]. We agree that extended lymph node dissection and adjuvant chemotherapies are beneficial for patients. We have performed D2 lymph node dissection for radical operations and adjuvant chemotherapies for the indicated patients in our center. In our study, there were no significant differences in the surgery type between the two groups. Although not statistically significant, curative resection increased by about $9.7 \%$ and postoperative complications decreased by about $8.7 \%$.

In our study, there was a statistically significant improvement in recurrence rate and survival rate in group II. The experience of the surgeon must have contributed to the survival rate. Although there was no statistically significant difference in cancer stage between 
the two groups, the patients in stage IV was considerably decreased. Moreover, the proportion of T4 that corresponded to advanced GC in tumor invasion was considerably decreased. Lymph node metastasis and distant metastasis were also statistically significant differences. There were significant differences in ASA scores between the two groups; low risk patients with low ASA scores in group II probably have affected survival improvement. However, because the two groups were categorized by time difference, the latter group naturally had relatively short follow-up duration. This should be taken into account when comparing the survival. From this point of view, we could not conclude that the recurrence rate and survival rate were clearly improved.

To summarize, our results are as follows: (1) group II had higher mean age than group I; (2) group II had lower ASA scores than group I; (3) the recurrence rate and survival rate showed statistically significant improvement in group II, however, it was not reliable due to the difference in follow-up duration; or (4) tumor depth, node metastasis and distant metastasis were significantly different between the two groups.

However, our results cannot be generalized because we retrospectively analyzed a small series of patients at a single, secondary medical institution. Our medical institution is a public hospital whose patients mainly include a socially underprivileged population, such as medical aid patients or the homeless, and it may show a difference from other common types of hospitals. The possibility of selection bias could not therefore be completely ruled out. This deserves further large-scale, multi-center studies.

Our study also has several advantages. All of the surgeries in this study were performed by a single surgeon. The strength of our study is that the surgical outcomes are not affected by operator variables. Our medical institution is a public hospital and differs from a general hospital in patient characteristics. It is meaningful to compare our results with other common types of hospitals.

In conclusion, our study showed the time dependent changes in clinico-pathologic characteristics and surgical outcomes in patients with GC. There were significant differences in the age, ASA score, average follow-up duration, postoperative hospital stay, tumor depth, node metastasis and distant metastasis between the two groups.

\section{CONFLICT OF INTEREST}

No potential conflict of interest relevant to this article was reported.

\section{REFERENCES}

1. Global Burden of Disease Cancer Collaboration, Fitzmaurice C, Allen C, Barber RM, Barregard L, Bhutta ZA, et al. Global, region- al, and national cancer incidence, mortality, years of life lost, years lived with disability, and disability-adjusted life-years for 32 cancer groups, 1990 to 2015: a systematic analysis for the global burden of disease study. JAMA Oncol 2017;3:524-48.

2. Rugge M, Genta RM, Di Mario F, El-Omar EM, El-Serag HB, Fassan $\mathrm{M}$, et al. Gastric cancer as preventable disease. Clin Gastroenterol Hepatol 2017;15:1833-43.

3. Choi IS, Choi M, Lee JH, Kim JH, Suh KJ, Lee JY, et al. Treatment patterns and outcomes in patients with metastatic gastric cancer receiving third-line chemotherapy: a population-based outcomes study. PLoS One 2018;13:e0198544.

4. Orditura M, Galizia G, Sforza V, Gambardella V, Fabozzi A, Laterza $\mathrm{MM}$, et al. Treatment of gastric cancer. World J Gastroenterol 2014;20:1635-49.

5. Lazar DC, Taban S, Cornianu M, Faur A, Goldis A. New advances in targeted gastric cancer treatment. World J Gastroenterol 2016; 22:6776-99.

6. Lin WL, Sun JL, Chang SC, Wu PH, Huang WT, Tsao CJ. Factors predicting survival of patients with gastric cancer. Asian Pac J Cancer Prev 2014;15:5835-8.

7. Song Z, Wu Y, Yang J, Yang D, Fang X. Progress in the treatment of advanced gastric cancer. Tumour Biol 2017;39:1010428317714626.

8. Bausys R, Bausys A, Vysniauskaite I, Maneikis K, Klimas D, Luksta $\mathrm{M}$, et al. Risk factors for lymph node metastasis in early gastric cancer patients: report from Eastern Europe country- Lithuania. BMC Surg 2017;17:108.

9. Marano L, Polom K, Patriti A, Roviello G, Falco G, Stracqualursi A, et al. Surgical management of advanced gastric cancer: an evolving issue. Eur J Surg Oncol 2016;42:18-27.

10. Japanese Gastric Cancer Association. Japanese gastric cancer treatment guidelines 2014 (ver. 4). Gastric Cancer 2017;20:1-19.

11. Borch K, Jonsson B, Tarpila E, Franzen T, Berglund J, Kullman E, et al. Changing pattern of histological type, location, stage and outcome of surgical treatment of gastric carcinoma. Br J Surg 2000;87: 618-26.

12. Information Committee of Korean Gastric Cancer Association. Korean Gastric Cancer Association nationwide survey on gastric cancer in 2014. J Gastric Cancer 2016;16:131-40.

13. Park IK, Oh HK, Park SH, Park JM. Short term outcomes of gastric cancer surgery in public hospital. Korean J Clin Oncol 2012;8:1622.

14. Kim H, Hwang Y, Sung H, Jang J, Ahn C, Kim SG, et al. Effectiveness of gastric cancer screening on gastric cancer incidence and mortality in a community-based prospective cohort. Cancer Res Treat 2018;50:582-9.

15. Craanen ME, Dekker W, Blok P, Ferwerda J, Tytgat GN. Time trends in gastric carcinoma: changing patterns of type and location. 
Am J Gastroenterol 1992;87:572-9.

16. Songun I, Putter H, Kranenbarg EM, Sasako M, van de Velde CJ. Surgical treatment of gastric cancer: 15-year follow-up results of the randomized nationwide Dutch D1D2 trial. Lancet Oncol 2010;11:439-49.

17. Tegels JJ, De Maat MF, Hulsewe KW, Hoofwijk AG, Stoot JH. Improving the outcomes in gastric cancer surgery. World J Gastroenterol 2014;20:13692-704.

18. Park CH, Song KY, Kim SN. Treatment results for gastric cancer surgery: 12 years' experience at a single institute in Korea. Eur J Surg Oncol 2008;34:36-41.

19. Ahn HS, Lee HJ, Yoo MW, Jeong SH, Park DJ, Kim HH, et al. Changes in clinicopathological features and survival after gastrectomy for gastric cancer over a 20-year period. Br J Surg 2011;98:255-60.

20. Lopez-Basave HN, Morales-Vasquez F, Ruiz-Molina JM, Namendys-Silva SA, Vela-Sarmiento I, Ruan JM, et al. Gastric cancer in young people under 30 years of age: worse prognosis, or delay in diagnosis? Cancer Manag Res 2013;5:31-6.

21. Saito H, Osaki T, Murakami D, Sakamoto T, Kanaji S, Tatebe S, et al. Effect of age on prognosis in patients with gastric cancer. ANZ J Surg 2006;76:458-61.

22. Blot WJ, Devesa SS, Kneller RW, Fraumeni JF Jr. Rising incidence of adenocarcinoma of the esophagus and gastric cardia. JAMA 1991;265:1287-9.

23. Devesa SS, Blot WJ, Fraumeni JF Jr. Changing patterns in the incidence of esophageal and gastric carcinoma in the United States.
Cancer 1998;83:2049-53.

24. Pernot S, Voron T, Perkins G, Lagorce-Pages C, Berger A, Taieb J. Signet-ring cell carcinoma of the stomach: impact on prognosis and specific therapeutic challenge. World J Gastroenterol 2015;21: 11428-38.

25. Miyahara R, Niwa Y, Matsuura T, Maeda O, Ando T, Ohmiya N, et al. Prevalence and prognosis of gastric cancer detected by screening in a large Japanese population: data from a single institute over 30 years. J Gastroenterol Hepatol 2007;22:1435-42.

26. Kubota H, Kotoh T, Masunaga R, Dhar DK, Shibakita M, Tachibana M, et al. Impact of screening survey of gastric cancer on clinicopathological features and survival: retrospective study at a single institution. Surgery 2000;128:41-7.

27. Maehara Y, Kakeji Y, Oda S, Takahashi I, Akazawa K, Sugimachi K. Time trends of surgical treatment and the prognosis for Japanese patients with gastric cancer. Br J Cancer 2000;83:986-91.

28. Wu CW, Hsiung CA, Lo SS, Hsieh MC, Chen JH, Li AF, et al. Nodal dissection for patients with gastric cancer: a randomised controlled trial. Lancet Oncol 2006;7:309-15.

29. Sasako M, Sano T, Yamamoto S, Kurokawa Y, Nashimoto A, Kurita A, et al. D2 lymphadenectomy alone or with para-aortic nodal dissection for gastric cancer. N Engl J Med 2008;359:453-62.

30. Chang SC, Liu KH, Hung CY, Tsai CY, Hsu JT, Yeh TS, et al. Adjuvant chemotherapy improves survival in stage III gastric cancer after D2 surgery. J Cancer 2018;9:81-91. 